\title{
Parotid Gland Carcinoma
}

National Cancer Institute

\section{Source}

National Cancer Institute. Parotid Gland Carcinoma. NCI Thesaurus. Code C6791.

A carcinoma that arises from the parotid gland. Representative examples include

mucoepidermoid carcinoma, adenoid cystic carcinoma, adenocarcinoma, and carcinoma ex pleomorphic adenoma. 